\title{
Strange duality revisited
}

\author{
Christian Pauly
}

We give a proof of the strange duality or rank-level duality of the WZW models of conformal blocks by extending the genus-0 result, obtained by Nakanishi-Tsuchiya in 1992, to higher genus curves via the sewing procedure. The new ingredient of the proof is an explicit use of the branching rules of the conformal embedding of affine Lie algebras $\widehat{\mathfrak{s l}(r)} \times \widehat{\mathfrak{s l}(l)} \subset \widehat{\mathfrak{s l}(r l)}$. We recover the strange duality of spaces of generalized theta functions obtained by Belkale, Marian-Oprea, as well as by Oudompheng in the parabolic case.

\section{Introduction}

One of the most significant recent results in the theory of vector bundles over curves is the proof of the strange duality or rank-level duality given by Belkale and Marian-Oprea. For a survey of the results we refer e.g., to [MO2, Pa2, Po]. In this note we give another proof of that duality in the framework of conformal blocks. In fact, the duality statement for conformal blocks over the projective line was proved by Nakanishi-Tsuchiya in 1992 $[\mathrm{NT}]$. We generalize their statement to a smooth projective curve of any genus.

In order to state the Main Theorem we need to introduce some notation. Let $r, l \geq 2$ be integers. We denote by $P_{l}(r)$ the finite set of dominant weights at level $l$ of the Lie algebra $\mathfrak{s l}(r)$ and for $\lambda \in P_{l}(r)$ we denote by $\mathcal{H}_{\lambda, l}(r)$ the irreducible integrable $\widehat{\mathfrak{s l}(r)}$-module of weight $\lambda$ and level $l$, where $\widehat{\mathfrak{s l}(r)}$ is the affine Lie algebra associated with $\mathfrak{s l}(r)$. The new ingredient of the proof is the use of the branching rules for the conformal embedding $\widehat{\mathfrak{s l}(r)} \times \widehat{\mathfrak{s l}(l)} \subset \widehat{\mathfrak{s l}(r l)}$ of the irreducible integrable $\widehat{\mathfrak{s l}(r l)}$-module $\mathcal{H}_{\lambda, 1}(r l)$, when $\lambda$ is a (possibly zero) fundamental dominant weight $\mathfrak{s l}(r l)$. The branching rule $[\mathrm{H}]$ gives the $81 \mathrm{~T} 40$

2000 Mathematics Subject Classification. Primary 14D20, 14H60, 17B67, 
decomposition

$$
\mathcal{H}_{\lambda, 1}(r l)=\bigoplus_{Y \in \mathcal{Y}_{r, l}^{\text {aff }}(\lambda)} \mathcal{H}_{\mu, l}(r) \otimes \mathcal{H}_{t} \mu, r(l)
$$

where $Y$ varies over a finite set $\mathcal{Y}_{r, l}^{\text {aff }}(\lambda) \subset \mathcal{Y}_{r, l}^{\text {aff }}$ of Young diagrams of type $(r, l)$ and of size $\lambda$ (for the definitions see Section 3.1). The dominant weights $\mu$ and ${ }^{t} \mu$ of $\mathfrak{s l}(r)$ and $\mathfrak{s l}(l)$ are naturally associated with $Y$ and to its transpose ${ }^{t} Y$. The above decomposition is an infinite-dimensional analogue of the classical Skew Cauchy Formula (see, e.g., [Pr]) giving the branching rule of the representation $\Lambda^{\lambda}\left(\mathbf{C}^{r} \otimes \mathbf{C}^{l}\right)$ for the embedding of finite Lie algebras $\mathfrak{s l}(r) \times \mathfrak{s l}(l) \subset \mathfrak{s l}(r l)$. Given a smooth projective curve $C$ with $n$ marked points and a collection $\vec{\lambda}=\left(\lambda_{1}, \ldots, \lambda_{n}\right) \in P_{l}(r)^{n}$ we denote by $\mathcal{V}_{\vec{\lambda}, l}^{\dagger}(C, r)$ the corresponding conformal block. With this notation we now can state the

Main Theorem. Let $C$ be a smooth projective complex curve with $n$ marked points and let

$$
\vec{\lambda}=\left(\lambda_{1}, \ldots, \lambda_{n}\right) \in P_{1}(r l)^{n}=\{0,1, \ldots, r l-1\}^{n}
$$

be a labelling of the marked points with fundamental weights of $\mathfrak{s l}(r l)$ satisfying the condition $\sum_{i=1}^{n} \lambda_{i} \equiv 0 \bmod r l$. For any collection of Young diagrams

$$
\vec{Y}=\left(Y_{1}, \ldots, Y_{n}\right) \in \prod_{i=1}^{n} \mathcal{Y}_{r, l}^{\mathrm{aff}}\left(\lambda_{i}\right)
$$

we denote by $\vec{\mu}=\pi(\vec{Y}) \in P_{l}(r)^{n}$ and ${ }^{t} \vec{\mu}=\pi\left({ }^{t} \vec{Y}\right) \in P_{r}(l)^{n}$ the collections of associated dominant weights of $\mathfrak{s l}(r)$ and $\mathfrak{s l}(l)$, respectively. Then the natural linear map between spaces of conformal blocks over the pointed curve $C$ obtained via the conformal embedding $\widehat{\mathfrak{s l}(r)} \times \widehat{\mathfrak{s l}(l)} \subset \overline{\mathfrak{s l}(r l)}$

$$
\alpha: \mathcal{V}_{\vec{\lambda}, 1}^{\dagger}(C, r l) \longrightarrow \mathcal{V}_{\vec{\mu}, l}^{\dagger}(C, r) \otimes \mathcal{V}_{t \vec{\mu}, r}^{\dagger}(C, l)
$$

induces an injective linear map

$$
S D_{\vec{Y}}: \mathcal{V}_{\vec{\mu}, l}(C, r) \longrightarrow \mathcal{V}_{\vec{\lambda}, 1}(C, r l) \otimes \mathcal{V}_{t \vec{\mu}, r}^{\dagger}(C, l)
$$

We recall that there is a canonical isomorphism (up to homothety) between the space of conformal blocks associated with a 1-pointed curve 
labelled with the trivial weight $\lambda_{1}=0$

$$
\mathcal{V}_{0, l}^{\dagger}(C, r) \cong H^{0}\left(\mathcal{S U}_{C}(r), \mathcal{L}^{l}\right)
$$

and the so-called space of generalized theta functions of rank $r$ and level $l$, i.e., the space of global sections of the $l$ th power of the determinant line bundle $\mathcal{L}$ over the coarse moduli space $\mathcal{S U}_{C}(r)$ of semi-stable rank- $r$ vector bundles with fixed trivial determinant over the curve $C$. In the special case of a 1-pointed curve labelled with the trivial weight and $Y_{1}=0, \mu_{1}=0,{ }^{t} \mu_{1}=0$ the above theorem combined with the isomorphism (1) states that the linear map

$$
S D_{0}: H^{0}\left(\mathcal{S U}_{C}(r), \mathcal{L}^{l}\right)^{\dagger} \longrightarrow H^{0}\left(\mathcal{S U}_{C}(r l), \mathcal{L}\right)^{\dagger} \otimes H^{0}\left(\mathcal{S U}_{C}(l), \mathcal{L}^{r}\right)
$$

is injective. We denote by $\mathcal{U}_{C}^{*}(r)$ the coarse moduli space of semi-stable rank $r$ and degree $r(g-1)$ vector bundles over $C$ and by $\Theta$ the divisor $\left\{E \in \mathcal{U}_{C}^{*}(r) \mid \operatorname{dim} H^{0}(C, E) \neq 0\right\} \subset \mathcal{U}_{C}^{*}(r)$. The tensor product map $\mathcal{U}_{C}^{*}(1) \times$ $\mathcal{S U}_{C}(l) \rightarrow \mathcal{U}_{C}^{*}(l)$ induces an inclusion

$$
\begin{aligned}
H^{0}\left(\mathcal{U}_{C}^{*}(l), \mathcal{O}(r \Theta)\right) & \subset H^{0}\left(\mathcal{U}_{C}^{*}(1), \mathcal{O}(r l \Theta)\right) \otimes H^{0}\left(\mathcal{S U}_{C}(l), \mathcal{L}^{r}\right) \\
& \cong H^{0}\left(\mathcal{S U}_{C}(r l), \mathcal{L}\right)^{\dagger} \otimes H^{0}\left(\mathcal{S U}_{C}(l), \mathcal{L}^{r}\right)
\end{aligned}
$$

The last isomorphism is proved in [BNR]. It is shown in [Be2] Lemma 2.1 (2) and Section 4.4 that the image of the linear map $S D_{0}$ is contained in $H^{0}\left(\mathcal{U}_{C}^{*}(l), \mathcal{O}(r \Theta)\right)$. Hence, assuming the well-known fact that both vector spaces have the same dimension, we obtain a new proof of the following theorem.

Theorem 1.1 ([Be1, MO1]). For any smooth curve $C$, the linear map

$$
S D_{0}: H^{0}\left(\mathcal{S U}_{C}(r), \mathcal{L}^{l}\right)^{\dagger} \longrightarrow H^{0}\left(\mathcal{U}_{C}^{*}(l), \mathcal{O}(r \Theta)\right)
$$

is an isomorphim.

Note the map $S D_{0}$ is defined here in terms of conformal blocks, but it coincides (see [Be2] Proposition 5.2) under the isomorphism (1) with the one defined at the level of moduli spaces of vector bundles.

The parabolic version of Theorem 1.1 proved by Oudompheng [O] Theorem 4.10 can be similarly deduced from our Main Theorem using the parabolic version of the isomorphism (1) proved in [Pa1]. We leave the details to the reader. 
The paper is organized as follows. In Sections 2-4, we collect for the reader's convenience some known results on conformal blocks, on the branching rules and on the Wess Zumino Witten (WZW)-connection. The proof of the Main Theorem is given in Section 5.

\section{Conformal blocks and factorization}

\subsection{Definition and properties of conformal blocks}

Given an integer $l \geq 1$ called the level, we introduce the finite set of dominant weights of $\mathfrak{s l}(r)$

$$
P_{l}(r)=\left\{\lambda=\sum_{i=1}^{r-1} a_{i} \varpi_{i} \mid \sum_{i=1}^{r-1} a_{i} \leq l ; a_{i} \geq 0\right\},
$$

where the $\varpi_{i}$ denote the $r-1$ fundamental weights of $\mathfrak{s l}(r)$. We also consider the involution of the set $P_{l}(r)$

$$
\lambda=\sum_{i=1}^{r-1} a_{i} \varpi_{i} \mapsto \lambda^{\dagger}=\sum_{i=1}^{r-1} a_{r-i} \varpi_{i}
$$

We denote by $V_{\lambda}$ the irreducible $\mathfrak{s l}(r)$-module with dominant weight $\lambda$. Then $\lambda^{\dagger}$ is the dominant weight of the dual $V_{\lambda}^{\dagger}$.

For the level $l=1$ we will often identify $P_{1}(r)$ with the set of integers $\{0,1, \ldots, r-1\}$ mapping the trivial weight to 0 and the $i$ th fundamental weight $\varpi_{i}$ to $i$. Under this identification the above involution (2) becomes $0^{\dagger}=0$ and $i^{\dagger}=r-i$ for $i>0$.

Given an integer $n \geq 1$, a collection $\vec{\lambda}=\left(\lambda_{1}, \ldots, \lambda_{n}\right) \in P_{l}(r)^{n}$ of dominant weights of $\mathfrak{s l}(r)$ and a family

$$
\mathcal{F}=\left(\pi: \mathcal{C} \rightarrow \mathcal{B} ; s_{1}, \ldots, s_{n} ; \xi_{1}, \ldots, \xi_{n}\right)
$$

of $n$-pointed stable curves of arithmetic genus $g$ parameterized by a base variety $\mathcal{B}$ with sections $s_{i}: \mathcal{B} \rightarrow \mathcal{C}$ and formal coordinates $\xi_{i}$ at the divisor $s_{i}(\mathcal{B}) \subset \mathcal{C}$, one constructs (see [TUY] Section 4.1) a locally free sheaf

$$
\mathcal{V}_{\vec{\lambda}, l}^{\dagger}(\mathcal{F}, r)
$$

over the base variety $\mathcal{B}$, called the sheaf of conformal blocks or the sheaf of vacua for the Lie algebra $\mathfrak{s l}(r)$ and the markings $\vec{\lambda}$ at level $l$. We recall 
that $\mathcal{V}_{\vec{\lambda}, l}^{\dagger}(\mathcal{F}, r)$ is a subsheaf of $\mathcal{O}_{\mathcal{B}} \otimes \mathcal{H}_{\vec{\lambda}, l}^{\dagger}$, where $\mathcal{H}_{\vec{\lambda}, l}^{\dagger}$ denotes the dual of the tensor product

$$
\mathcal{H}_{\vec{\lambda}, l}=\mathcal{H}_{\lambda_{1}, l} \otimes \cdots \otimes \mathcal{H}_{\lambda_{n}, l}
$$

of the integrable highest weight representations $\mathcal{H}_{\lambda_{i}, l}$ of level $l$ and weight $\lambda_{i}$ of the affine Lie algebra $\widehat{\mathfrak{s l}(r)}$. The formation of the sheaf of conformal blocks commutes with base change. In particular, we have for any point $b \in \mathcal{B}$

$$
\mathcal{V}_{\vec{\lambda}, l}^{\dagger}(\mathcal{F}, r) \otimes_{\mathcal{O}_{\mathcal{B}}} \mathcal{O}_{b} \cong \mathcal{V}_{\vec{\lambda}, l}^{\dagger}(C, r)
$$

where $C$ denotes the data $\left(\mathcal{C}_{b}=\pi^{-1}(b) ; s_{1}(b), \ldots, s_{n}(b) ; \xi_{1 \mid \mathcal{C}_{b}}, \ldots, \xi_{n \mid \mathcal{C}_{b}}\right)$ consisting of a stable curve $\mathcal{C}_{b}$ with $n$ marked points $s_{1}(b), \ldots, s_{n}(b)$ and formal coordinates $\xi_{i \mid \mathcal{C}_{b}}$ at the points $s_{i}(b)$.

We recall that the sheaf of conformal blocks $\mathcal{V}_{\vec{\lambda}, l}^{\dagger}(\mathcal{F}, r)$ does not depend (up to a canonical isomorphism) on the formal coordinates $\xi_{i}$ (see, e.g., [U] Theorem 4.1.7). We therefore omit the formal coordinates in the notation.

We have the following factorization theorem.

Proposition 2.1 ([TUY]). Let $C$ be a nodal curve with a node $n$ and let $\pi: \widetilde{C} \rightarrow C$ be the partial desingularization at $n$. Then we have the direct sum decomposition

$$
\mathcal{V}_{\vec{\lambda}, l}^{\dagger}(C, r)=\bigoplus_{\mu \in P_{l}(r)} \mathcal{V}_{\vec{\lambda} \cup \mu \cup \mu^{\dagger}, l}^{\dagger}(\widetilde{C}, r)
$$

where we put the weights $\mu$ and $\mu^{\dagger}$ at the two points $a, b \in \widetilde{C}$ lying over the node $n \in C$.

Lemma 2.2. Let $C$ be a stable curve of genus $g$ with $n$ marked points labelled with the dominant weights $\vec{\lambda}=\left(\lambda_{1}, \ldots, \lambda_{n}\right) \in P_{1}(r l)^{n}$. Then

$$
\operatorname{dim} \mathcal{V}_{\vec{\lambda}, 1}^{\dagger}(C, r l)= \begin{cases}(r l)^{g} & \text { if } \sum_{i=1}^{n} \lambda_{i} \equiv 0 \bmod r l \\ 0 & \text { otherwise }\end{cases}
$$

Proof. This is a straightforward consequence of the factorization of conformal blocks when degenerating the genus- $g$ curve to a rational curve with $g$ nodes and taking the desingularization — we iterate Proposition $2.1 \mathrm{~g}$ times. 
The formula states that

$$
\operatorname{dim} \mathcal{V}_{\vec{\lambda}, 1}^{\dagger}(C, r l)=\sum_{\vec{\mu} \in P_{1}(r l)^{g}} \operatorname{dim} \mathcal{V}_{\vec{\lambda} \cup \vec{\mu} \cup \vec{\mu}^{\dagger}, 1}^{\dagger}\left(\mathbf{P}^{1}, r l\right) .
$$

On the other hand, $\sum_{i=1}^{n} \lambda_{i}+\sum_{j=1}^{g} \mu_{j}+\mu_{j}^{\dagger} \equiv \sum_{i=1}^{n} \lambda_{i} \bmod r l$, since $\mu_{j}+$ $\mu_{j}^{\dagger} \equiv 0 \bmod r l$. Thus, it is sufficient to prove the dimension formula for $g=0$. Then use once more the factorization formula to reduce to the case of $\mathbf{P}^{1}$ with three marked points, i.e., $g=0$ and $n=3$. That calculation is standard, see e.g., [G] Formula 2.14 or [NT] Section 4.

\section{Branching rules}

In this section, we review the results on the branching rules of $[\mathrm{H}]$ used in the proof of the Main Theorem.

\subsection{Young diagrams}

Given two positive integers $r$ and $l$, we will denote by Young diagram of type $(r, l)$ a decreasing sequence of $r$ positive integers

$$
Y=\left(y_{1} \geq y_{2} \geq \cdots \geq y_{r-1} \geq y_{r} \geq 0\right) \text { such that } y_{1}-y_{r} \leq l .
$$

We denote the (infinite) set of Young diagrams of type $(r, l)$ by $\mathcal{Y}_{r, l}$ and consider the map to the set of dominant weights of $\mathfrak{s l}(r)$

$$
\pi: \mathcal{Y}_{r, l} \longrightarrow P_{l}(r), \quad Y \mapsto \pi(Y)=\sum_{i=1}^{r-1}\left(y_{i}-y_{i+1}\right) \varpi_{i} .
$$

We introduce the finite subsets:

$$
\mathcal{Y}_{r, l}^{\text {aff }}=\left\{Y \in \mathcal{Y}_{r, l} \mid y_{r} \leq l-1\right\} \quad \text { and } \quad \mathcal{Y}_{r, l}^{\text {fin }}=\left\{Y \in \mathcal{Y}_{r, l}^{\text {aff }} \mid y_{1} \leq l\right\} .
$$

Note that all fibres of the map $\pi: \mathcal{Y}_{r, l}^{\text {aff }} \longrightarrow P_{l}(r)$ have cardinality $l$.

We will now define several maps between these finite sets of Young diagrams. We can think of a Young diagram of type $(r, l)$ as a collection of $r$ rows, where we put into the $i$ th row $y_{i}$ boxes. We distinguish two cases:

Case 1: $Y \in \mathcal{Y}_{r, l}^{\text {fin }}$. We have $y_{1} \leq l$ and $y_{r} \leq l-1$. We put ${ }^{t} Y \in \mathcal{Y}_{l, r}^{\text {fin }}$ the Young diagram of size $(l, r)$ defined by taking the transpose of $Y$, i.e., by putting the $y_{i}$ boxes into the $i$ th column. For $Y \neq(0, \ldots, 0)$ we define $Y^{\dagger} \in$ 
$\mathcal{Y}_{r, l}^{\text {fin }}$ to be the complement (after a $180^{\circ}$ rotation) of $Y$ in the full rectangle consisting of $r$ rows having $l$ boxes each, and for $Y=(0, \ldots, 0)$ we define $Y^{\dagger}=Y$.

Case 2: $Y \in \mathcal{Y}_{r, l}^{\text {aff }} \backslash \mathcal{Y}_{r, l}^{\text {fin }}$. We have $l+1 \leq y_{1} \leq 2 l-1$ and $y_{r} \leq l-1$. We can write the Young diagram $Y$ as a union of two diagrams $Y_{1} \cup Y_{2}$ with $Y_{i} \in \mathcal{Y}_{r, l}^{\text {fin }}$, where the first one $Y_{1}$ has in its $i$ th row $\min \left(l, y_{i}\right)$ boxes and the second one $Y_{2}$ has in its $i$ th row $\max \left(y_{i}-l, 0\right)$ boxes. We then define the transpose ${ }^{t} Y$ to be the union of the transposes ${ }^{t} Y_{1} \cup{ }^{t} Y_{2}$, where the ${ }^{t} Y_{i}$ are defined as in Case 1. Note that the Young diagram fits into two full rectangles consisting of $r$ rows having $2 l$ boxes each. We then define $Y^{\dagger} \in \mathcal{Y}_{r, l}^{\text {aff }}$ to be the complement (after a 180 degree rotation) of $Y$ in that double rectangle.

We thus have constructed two involutive bijective maps

$$
\begin{aligned}
& \mathcal{Y}_{r, l}^{\text {aff }} \longrightarrow \mathcal{Y}_{r, l}^{\text {aff }} \quad \text { and } \quad \begin{aligned}
\mathcal{Y}_{r, l}^{\text {aff }} & \longrightarrow \mathcal{Y}_{l, r}^{\text {aff }} \\
Y & \mapsto Y^{\dagger}
\end{aligned} \quad \text { } \\
& Y{ }^{t} Y
\end{aligned}
$$

These two maps preserve the subsets $\mathcal{Y}_{r, l}^{\mathrm{fin}} \subset \mathcal{Y}_{r, l}^{\mathrm{aff}}$ and $\mathcal{Y}_{l, r}^{\mathrm{fin}} \subset \mathcal{Y}_{l, r}^{\mathrm{aff}}$. Note that ${ }^{t}\left(Y^{\dagger}\right)=\left({ }^{t} Y\right)^{\dagger}$ and that $\pi\left(Y^{\dagger}\right)=\pi(Y)^{\dagger}$, where $\pi(Y)^{\dagger}$ is defined as in (2). We also note the equalities

$$
\left|\mathcal{Y}_{r, l}^{\mathrm{aff}}\right|=\left|\mathcal{Y}_{l, r}^{\mathrm{aff}}\right|=l\left|P_{l}(r)\right|=r\left|P_{r}(l)\right|
$$

Given a Young diagram $Y \in \mathcal{Y}_{r, l}$ we define its size $|Y|=\sum_{i=1}^{r} y_{i} \bmod r l$. Moreover, we identify $\mathbf{Z} / r l \mathbf{Z}=\{0, \ldots, r l-1\}$, so that $|Y| \in\{0, \ldots, r l-$ $1\}$. It is clear from the definition that $\left|Y^{\dagger}\right|+|Y|=r l$ and that $\left|{ }^{t} Y\right|=|Y|$. We will denote by $\mathcal{Y}_{r, l}^{\text {fin }}(\lambda)$ and $\mathcal{Y}_{r, l}^{\text {aff }}(\lambda)$ the corresponding subsets of Young diagrams of size $\lambda$.

Example 3.1. We consider the following example for $r=3$ and $l=4$ : $Y=(6,4,3) \in \mathcal{Y}_{3,4}^{\text {aff }} \backslash \mathcal{Y}_{3,4}^{\text {fin }}$. Then we have the following table.

$$
Y=(6,4,3) \quad{ }^{t} Y=(4,4,3,2) \quad Y^{\dagger}=(5,4,2) \quad{ }^{t} Y^{\dagger}=(4,3,2,2)
$$
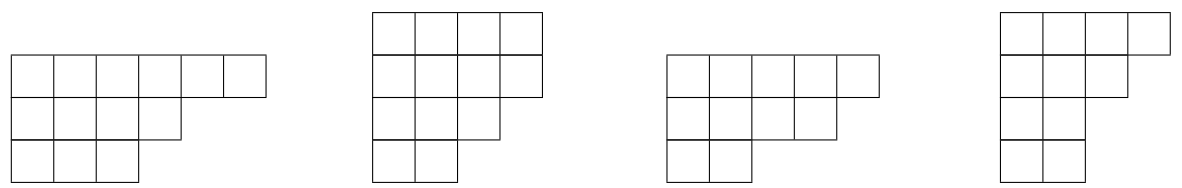

$$
\pi(Y)=2 \varpi_{1}+\varpi_{2} \pi\left(Y^{\dagger}\right)=\varpi_{1}+2 \varpi_{2} \pi\left({ }^{t} Y\right)=\varpi_{2}+\varpi_{3} \pi\left({ }^{t} Y^{\dagger}\right)=\varpi_{1}+\varpi_{2}
$$

Moreover $|Y|=\left|{ }^{t} Y\right|=1$ and $\left|Y^{\dagger}\right|=\left|{ }^{t} Y^{\dagger}\right|=11$. 


\subsection{Finite-dimensional case}

We now recall the classical Skew Cauchy Formula (see e.g., [Pr] Theorem 8.4.1. Chapter 9), which gives the branching rule of the fundamental $\mathfrak{s l}(r l)$-modules under the embedding $\mathfrak{s l}(r) \times \mathfrak{s l}(l) \subset \mathfrak{s l}(r l)$. Let $\lambda$ be in $\{0, \ldots, r l-1\}$. Under the identification $\{0, \ldots, r l-1\}=P_{1}(r l)$ the $\mathfrak{s l}(r l)$-module $\Lambda^{\lambda} \mathbf{C}^{r l}=\Lambda^{\lambda}\left(\mathbf{C}^{r} \otimes \mathbf{C}^{l}\right)$ corresponds to the fundamental weight $\lambda \in P_{1}(r l)$ and decomposes as sum of irreducible $\mathfrak{s l}(r) \times \mathfrak{s l}(l)$-modules

$$
V_{\lambda}=\Lambda^{\lambda}\left(\mathbf{C}^{r} \otimes \mathbf{C}^{l}\right)=\bigoplus_{Y \in \mathcal{Y}_{r, l}^{\mathrm{fin}}(\lambda)} V_{\mu} \otimes V_{t} \mu
$$

where $V_{\mu}$ and $V_{t}$ denote the $\mathfrak{s l}(r)$ and $\mathfrak{s l}(l)$-modules with dominant weights $\mu=\pi(Y) \in P_{l}(r)$ and ${ }^{t} \mu=\pi\left({ }^{t} Y\right) \in P_{r}(l)$.

\subsection{Infinite-dimensional case}

The analogue of the above Skew Cauchy Formula for the embedding of affine Lie algebras $\widehat{\mathfrak{s l}(r)} \times \widehat{\mathfrak{s l}(l)} \subset \widehat{\mathfrak{s l}(r l)}$ is worked out in [H] Theorem 4.2. With the above notation we have the decomposition as sum of irreducible $\widehat{\mathfrak{s l}(r)} \times \widehat{\mathfrak{s l}(l)}$-modules

$$
\mathcal{H}_{\lambda, 1}=\bigoplus_{Y \in \mathcal{Y}_{r, l}^{\text {aff }}(\lambda)} \mathcal{H}_{\mu, l} \otimes \mathcal{H}_{t} \mu, r
$$

The Virasoro operator $L_{0}$ associated with $\mathfrak{s l}(r l)$ induces a decomposition (see $[\mathrm{TUY}]$ or $[\mathrm{U}]$ ) into eigenspaces of the $\widehat{\mathfrak{s l}(r l)}$-module

$$
\mathcal{H}_{\lambda, 1}=\bigoplus_{d=0}^{\infty} \mathcal{H}_{\lambda, 1}(d) \quad \text { with } \quad \mathcal{H}_{\lambda, 1}(0)=V_{\lambda}
$$

For every Young diagram $Y \in \mathcal{Y}_{r, l}^{\text {aff }}(\lambda)$ we have an inclusion

$$
\mathcal{H}_{\mu, l} \otimes \mathcal{H}_{t} \mu, r \hookrightarrow \mathcal{H}_{\lambda, 1}
$$

Note that the Virasoro operators $L_{0}$ associated with the two Lie algebras $\mathfrak{s l}(r) \times \mathfrak{s l}(l) \subset \mathfrak{s l}(r l)$ coincide since these Lie algebras form a conformal pair (see $[\mathrm{KW}]$ Proposition $3.2(\mathrm{c})$ ). Hence, restricting the previous inclusion to 
the 0-eigenspace we obtain an inclusion

$$
\mathcal{H}_{\mu, l} \otimes \mathcal{H}^{t} \mu, r(0)=V_{\mu} \otimes V_{t}{ }_{\mu} \hookrightarrow \mathcal{H}_{\lambda, 1}\left(n_{Y}\right)
$$

for some positive integer $n_{Y}$. It follows from the Skew Cauchy Formula (4) that

$$
Y \in \mathcal{Y}_{r, l}^{\mathrm{fin}}(\lambda) \Longleftrightarrow n_{Y}=0
$$

\section{The projective WZW-connection}

\subsection{Definition of the projective WZW-connection}

We now outline the definition of the projective WZW-connection on the sheaf $\mathcal{V}_{\vec{\lambda}, l}^{\dagger}(\mathcal{F}, r)$ over the smooth locus $\mathcal{B}^{s} \subset \mathcal{B}$ parameterizing smooth curves and refer to [TUY] or [U] for a detailed account. Let $\mathcal{D} \subset \mathcal{B}$ be the discriminant locus and let $\mathcal{S}=\coprod_{i=1}^{n} s_{i}(\mathcal{B})$ be the union of the images of the $n$ sections. We recall the exact sequence

$$
0 \longrightarrow \pi_{*} \Theta_{\mathcal{C} / \mathcal{B}}(* \mathcal{S}) \longrightarrow \pi_{*} \Theta_{\mathcal{C}}^{\prime}(* \mathcal{S})_{\pi} \stackrel{\theta}{\longrightarrow} \Theta_{\mathcal{B}}(-\log \mathcal{D}) \longrightarrow 0
$$

where $\Theta_{\mathcal{C} / \mathcal{B}}(* \mathcal{S})$ denotes the sheaf of vertical rational vector fields on $\mathcal{C}$ with poles only along the divisor $\mathcal{S}$, and $\Theta_{\mathcal{C}}^{\prime}(* \mathcal{S})_{\pi}$ the sheaf of rational vector fields on $\mathcal{C}$ with poles only along the divisor $\mathcal{S}$ and with constant horizontal components along the fibres of $\pi$. There is an $\mathcal{O}_{\mathcal{B}}$-linear map

$$
p: \pi_{*} \Theta_{\mathcal{C}}^{\prime}(* \mathcal{S})_{\pi} \longrightarrow \bigoplus_{i=1}^{n} \mathcal{O}_{\mathcal{B}}\left(\left(\xi_{i}\right)\right) \frac{d}{d \xi_{i}}
$$

which associates with a vector field $\vec{\ell}$ in $\Theta_{\mathcal{C}}^{\prime}(* \mathcal{S})_{\pi}$ the $n$ Laurent expansions $\ell_{i} \frac{d}{d \xi_{i}}$ around the divisor $s_{i}(\mathcal{B})$. Abusing notation we also write $\vec{\ell}$ for its image under $p$

$$
\vec{\ell}=\left(\ell_{1} \frac{d}{d \xi_{1}}, \ldots, \ell_{n} \frac{d}{d \xi_{n}}\right) \in \bigoplus_{i=1}^{n} \mathcal{O}_{\mathcal{B}}\left(\left(\xi_{i}\right)\right) \frac{d}{d \xi_{i}} .
$$

We then define for any vector field $\vec{\ell}$ in $\Theta_{\mathcal{C}}^{\prime}(* \mathcal{S})_{\pi}$ the endomorphism $D(\vec{\ell})$ of $\mathcal{O}_{\mathcal{B}} \otimes \mathcal{H}_{\vec{\lambda}, l}^{\dagger}$ by

$$
D(\vec{\ell})(f \otimes u)=\theta(\vec{\ell}) . f \otimes u+\sum_{i=1}^{n} f \otimes\left(T\left[\ell_{i}\right] . u\right)
$$


for $f$ a local section of $\mathcal{O}_{\mathcal{B}}$ and $u \in \mathcal{H}_{\vec{\lambda}, l}^{\dagger}$. Here $T\left[\ell_{i}\right]$ denotes the action of the energy-momentum tensor on the $i$ th component $\mathcal{H}_{\lambda_{i}, l}^{\dagger}$, i.e., expanding $\ell_{i}=\sum_{j=-n_{0}}^{\infty} \alpha_{j} \xi_{i}^{j+1}$ with $\alpha_{j}$ local sections of $\mathcal{O}_{\mathcal{B}}$, the operator $T\left[\ell_{i}\right]$ equals $\sum_{j=-n_{0}}^{\infty} \alpha_{j} L_{j}$. Here the operators $L_{j}$ are the Virasoro operator acting linearly on $\mathcal{H}_{\lambda_{i}, l}^{\dagger}$ via the Sugawara representation. It is shown in [TUY] that $D(\vec{\ell})$ preserves $\mathcal{V}_{\vec{\lambda}, l}^{\dagger}(\mathcal{F}, r)$ and that $D(\vec{\ell})$ only depends on the image $\theta(\vec{\ell})$ up to homothety. One therefore obtains a projective connection $\nabla$ on the sheaf $\mathcal{V}_{\vec{\lambda}, l}^{\dagger}(\mathcal{F}, r)$ over $\mathcal{B}^{s}$ given by

$$
\nabla_{\theta(\vec{\ell})}=\theta(\vec{\ell})+T[\vec{\ell}]
$$

\subsection{Conformal embedding and projective flatness}

Proposition 4.1. Let $\mathcal{F}$ be a family of smooth projective n-pointed curves as in (3) and let $\vec{\lambda} \in P_{1}(r l)^{n}$ be a labelling of the marked points with fundamental weights of $\mathfrak{s l}(r l)$. Then for every collection of Young diagrams $\vec{Y}=\left(Y_{1}, \ldots, Y_{n}\right) \in \prod_{i=1}^{n} \mathcal{Y}_{r, l}^{\text {aff }}\left(\lambda_{i}\right)$ the tensor product of the $n$ inclusions (5)

$$
\mathcal{H}_{\vec{\mu}, l} \otimes \mathcal{H}_{t \vec{\mu}, r} \hookrightarrow \mathcal{H}_{\vec{\lambda}, 1}
$$

induces a natural homomorphism between sheaves of conformal blocks

$$
\left(\mathcal{V}_{\vec{\lambda}, 1}^{\dagger}(\mathcal{F}, r l), \nabla\right) \longrightarrow\left(\mathcal{V}_{\vec{\mu}, l}^{\dagger}(\mathcal{F}, r), \nabla\right) \otimes\left(\mathcal{V}_{t \vec{\mu}, r}^{\dagger}(\mathcal{F}, l), \nabla\right),
$$

which is projectively flat for the WZW connections.

Proof. We consider the embedding of semi-simple Lie algebras $\mathfrak{p}=\mathfrak{s t}(r) \times$ $\mathfrak{s l}(l) \subset \mathfrak{g}=\mathfrak{s l}(r l)$. Since this embedding is conformal, we have by [KW] Proposition 3.2(c) that for any integer $n$ the two Virasoro operators $L_{n}$ associated with $\mathfrak{p}$ and $\mathfrak{g}$ coincide. The proposition now follows since the two linear parts of the connections (7) given by the energy-momentum tensor also coincide.

Remark 4.2. Note that the WZW-connection is only defined for a family of smooth curves.

Remark 4.3. The previous proposition actually holds for any pair $\mathfrak{p} \subset \mathfrak{g}$ of conformal embeddings of semi-simple Lie algebras. For the list of conformal embeddings, see e.g., [BB]. 
We have the following

Corollary 4.4. With the above notation the homomorphism obtained from (8)

$$
S D_{\vec{Y}}:\left(\mathcal{V}_{\vec{\mu}, l}(\mathcal{F}, r), \nabla\right) \longrightarrow\left(\mathcal{V}_{\vec{\lambda}, 1}(\mathcal{F}, r l), \nabla\right) \otimes\left(\mathcal{V}_{t \vec{\mu}, r}^{\dagger}(\mathcal{F}, l), \nabla\right)
$$

is projectively flat and has constant rank.

Proof. Both assertions are valid for any vector bundles equipped with projective connections. We refer, e.g., to [Be2] Lemmas A.1 and A.2 for a proof.

\section{Proof of the Main Theorem}

We prove the Main Theorem by induction on the genus $g$ of the curve. For $g=0$ the Theorem coincides with $[\mathrm{NT}]$ Theorem 4.4, since in that case $\operatorname{dim} \mathcal{V}_{\vec{\lambda}, 1}(C, r l)=1$ by Lemma 2.2. Note that for $g=0$ the map $S D_{\vec{Y}}$ is an isomorphism.

We now assume that the Theorem holds for any smooth marked curve of genus $g-1$. First of all we notice that the map $S D_{\vec{Y}}$ as defined in the Main Theorem for smooth curves can be defined as well for a nodal curve. Next, we observe that it is enough to show injectivity of the map $S D_{\vec{Y}}$ for a curve $C$ of genus $g$ with one node. In fact, by upper semi-continuity of the rank of a homomorphism between vector bundles, we then obtain injectivity of $S D_{\vec{Y}}$ for a general smooth curve. Then, since the rank of $S D_{\vec{Y}}$ is constant for smooth families, as shown in Corollary 4.4, we obtain injectivity for any smooth curve.

We consider the desingularization $\pi: \widetilde{C} \rightarrow C$ of the nodal curve $C$ of genus $g$. Note that $\widetilde{C}$ is smooth of genus $g-1$. The linear map $\alpha$ decomposes under the factorization given by Proposition 2.1 as follows:

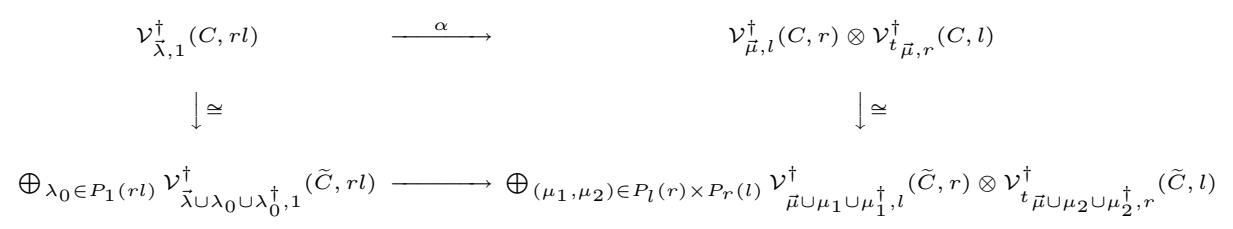

For any triple $\left(\lambda_{0}, \mu_{1}, \mu_{2}\right) \in P_{1}(r l) \times P_{l}(r) \times P_{r}(l)$ we define the map

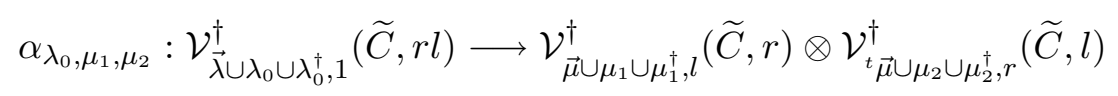

as the $\left(\lambda_{0}, \mu_{1}, \mu_{2}\right)$ component of $\alpha$ in the above decomposition. 
Definition 5.1. We say that a triple $\left(\lambda_{0}, \mu_{1}, \mu_{2}\right) \in P_{1}(r l) \times P_{l}(r) \times P_{r}(l)$ is admissible if there exists a Young diagram $Y \in \mathcal{Y}_{r, l}^{\text {aff }}\left(\lambda_{0}\right)$ such that $\pi(Y)=$ $\mu_{1} \in P_{l}(r)$ and $\pi\left({ }^{t} Y\right)=\mu_{2} \in P_{r}(l)$.

It is clear from the definition that if $\left(\lambda_{0}, \mu_{1}, \mu_{2}\right)$ is admissible then the corresponding Young diagram $Y \in \mathcal{Y}_{r, l}^{\text {aff }}\left(\lambda_{0}\right)$ is uniquely determined either by the pair $\left(\lambda_{0}, \mu_{1}\right)$ or by the pair $\left(\lambda_{0}, \mu_{2}\right)$.

We now recall [BP] Proposition 4.4 in our context, i.e., for the conformal embedding $\mathfrak{p}=\mathfrak{s l}(r) \times \mathfrak{s l}(l) \subset \mathfrak{g}=\mathfrak{s l}(r l)$.

Proposition 5.2. Given a triple $\left(\lambda_{0}, \mu_{1}, \mu_{2}\right) \in P_{1}(r l) \times P_{l}(r) \times P_{r}(l)$ the linear map $\alpha_{\lambda_{0}, \mu_{1}, \mu_{2}}$ is

- identically zero, if $\left(\lambda_{0}, \mu_{1}, \mu_{2}\right)$ is not admissible, or if $\left(\lambda_{0}, \mu_{1}, \mu_{2}\right)$ is admissible and $Y \in \mathcal{Y}_{r, l}^{\text {aff }}\left(\lambda_{0}\right) \backslash \mathcal{Y}_{r, l}^{\text {fin }}\left(\lambda_{0}\right)$

- is induced by the natural inclusion

$$
\begin{aligned}
& \mathcal{H}_{\vec{\mu}, l} \otimes \mathcal{H}_{t} \vec{\mu}, r \\
& \text { if }\left(\lambda_{0}, \mu_{1}, \mu_{2}\right) \text { is admissible and } Y \in \mathcal{H}_{\mu_{1}, l} \otimes \mathcal{H}_{\mu_{2}, r} \otimes \mathcal{H}_{\mu_{1}^{\dagger}, l} \otimes \mathcal{H}_{\mu_{2}^{\dagger}, r} \hookrightarrow \mathcal{H}_{\vec{\lambda}, 1} \otimes \mathcal{H}_{\lambda_{0}, 1} \otimes \mathcal{H}_{\lambda_{0}^{\dagger}, 1},
\end{aligned}
$$

We denote by

$$
S D\left(\lambda_{0}, \mu_{1}, \mu_{2}\right): \mathcal{V}_{\vec{\mu} \cup \mu_{1} \cup \mu_{1}^{\dagger}, l}(\widetilde{C}, r) \longrightarrow \mathcal{V}_{\vec{\lambda} \cup \lambda_{0} \cup \lambda_{0}^{\dagger}, 1}(\widetilde{C}, r l) \otimes \mathcal{V}_{t \vec{\mu} \cup \mu_{2} \cup \mu_{2}^{\dagger}, r}^{\dagger}(\widetilde{C}, l)
$$

the linear map induced by $\alpha_{\lambda_{0}, \mu_{1}, \mu_{2}}$. If we are in the second case of Proposition 5.2, i.e., if $\left(\lambda_{0}, \mu_{1}, \mu_{2}\right)$ is admissible and $Y \in \mathcal{Y}_{r, l}^{\mathrm{fin}}\left(\lambda_{0}\right)$, then Proposition 5.2 and the induction hypothesis applied to the curve $\widetilde{C}$ implies that the map $S D\left(\lambda_{0}, \mu_{1}, \mu_{2}\right)$ is injective. In all other cases, this map is zero. Now we observe that for any pair $\left(\lambda_{0}, \mu_{2}\right) \in P_{1}(r l) \times P_{r}(l)$ there exists at most one $\mu_{1} \in P_{l}(r)$ such that the triple $\left(\lambda_{0}, \mu_{1}, \mu_{2}\right)$ is admissible. We introduce the set $S\left(\mu_{1}\right)=\left\{\left(\lambda_{0}, \mu_{2}\right) \mid\left(\lambda_{0}, \mu_{1}, \mu_{2}\right)\right.$ admissible $\}$. In order to show injectivity of $S D_{\vec{Y}}$ it is enough to show for each $\mu_{1} \in P_{l}(r)$ injectivity of the map

$$
\mathcal{V}_{\vec{\mu} \cup \mu_{1} \cup \mu_{1}^{\dagger}, l}(\widetilde{C}, r) \longrightarrow \bigoplus_{\left(\lambda_{0}, \mu_{2}\right) \in S\left(\mu_{1}\right)} \mathcal{V}_{\vec{\lambda} \cup \lambda_{0} \cup \lambda_{0}^{\dagger}, 1}(\widetilde{C}, r l) \otimes \mathcal{V}_{t \vec{\mu} \cup \mu_{2} \cup \mu_{2}^{\dagger}, r}^{\dagger}(\widetilde{C}, l) .
$$

But this follows from the fact that the map $S D\left(\lambda_{0}, \mu_{1}, \mu_{2}\right)$ is injective for an admissible triple with $Y \in \mathcal{Y}_{r, l}^{\text {fin }}\left(\lambda_{0}\right)$. Since $\pi: \mathcal{Y}_{r, l}^{\text {fin }} \rightarrow P_{l}(r)$ is surjective, we can find for each $\mu_{1} \in P_{l}(r)$ such a pair $\left(\lambda_{0}, \mu_{2}\right) \in S\left(\mu_{1}\right)$. This completes the proof. 


\section{Acknowledgements}

I would like to thank Laurent Manivel for helpful comments. This research was supported by a Marie Curie Intra European Fellowship within the 7th European Community Framework Programme.

\section{References}

[BB] A. Bais and P. Bouwknegt, A classification of subgroup truncations of the bosonic string, Nucl. Phys. B 279 (1987), 561-570.

[BNR] A. Beauville, M.S. Narasimhan and S. Ramanan, Spectral curves and the generalised theta divisor, J. Reine Angew. Math. 398 (1989), 169-179.

[Be1] P. Belkale, The strange duality conjecture for generic curves, J. Amer. Math. Soc. 21 (2008), 235-258.

[Be2] Strange duality and the Hitchin/WZW connection, J. Differ. Geom. 82(2) (2009), 445-465.

[BP] A. Boysal and C. Pauly, Strange duality for Verlinde spaces of exceptional groups at level one, Int. Math. Res. Not. 4 (2010), 595-618.

[G] D. Gepner, Fusion rings and geometry, Commun. Math. Phys. 141 (1991), 381-411.

[H] K. Hasegawa, Spin module versions of Weyl's reciprocity theorem for classical Kac-Moody Lie algebras - an application to branching rule duality, Publ. Res. Inst. Math. Sci. 25 (1989), 741-828.

[KW] V. Kac and M. Wakimoto, Modular and conformal invariance constraints in representation theory of affine algebras, Adv. Math. 70 (1988), 156-234.

[MO1] A. Marian and D. Oprea, The level-rank duality for non-abelian theta functions, Invent. Math. 168(2) (2007), 225-247.

[MO2] _ A tour of theta dualities on moduli spaces of sheaves, Curves and abelian varieties, 175-202, Contemporary Mathematics, 465, American Mathematical Society, Providence, Rhode Island, 2008.

[NT] T. Nakanishi and A. Tsuchiya, Level-rank duality of WZW models in conformal field theory, Commun. Math. Phys. 144 (1992), 351-372. 
[O] R. Oudompheng, Rank-level duality for conformal blocks of the linear group, J. Algebraic Geom. 20 (2011), 559-597.

[Pa1] C. Pauly, Espaces de modules de fibrés paraboliques et blocs conformes, Duke Math. J. 86(1) (1996), 217-235.

[Pa2] _ La dualité étrange, Séminaire Bourbaki, Vol. 2007/2008, Astérisque No. 326 (2009), Exp. No. 994, 363-377.

[Po] M. Popa, Generalized theta linear series on moduli spaces of vector bundles on curves, 'Handbook of Moduli', (G. Farkas and I. Morrison eds.), Vol. III, 219-255. Adv. Lect. Math. (ALM), 26, Int. Press, Somerville, MA, 2013.

[Pr] C. Procesi, Lie Groups, An Approach through Invariants and Representations. Universitext, Springer, 2007.

[TUY] A. Tsuchiya, K. Ueno and Y. Yamada, Conformal field theory on universal family of stable curves with gauge symmetries, Advanced Studies in Pure Mathematics 19, 1989, Kinokuniya Shoten and Academic Press, 459-566.

[U] K. Ueno, Introduction to conformal field theory with gauge symmetries, in 'Geometry and Physics', Lecture Notes in Pure and Applied Mathematics, 184, Marcel Dekker, 1996, 603-745.

Laboratoire de Mathématiques J. A. Dieudonné

Université DE NiCE - Sophia Antipolis

06108 Nice Cedex 02

FRANCE

E-mail address: pauly@unice.fr

Received April 25, 2012 